\title{
Estimating risk and cost: An analysis of patients with risk factors for Type 2 Diabetes in rural Jalisco, Mexico
}

Katie Morales, Emily Hall, Ella Harris, Alden Blair, Sharon Rose, Sergio Bautista-Arredondo, Nicole Santos, Martin Sandoval, Alberto Tovar, Kimberly Baltzell

University of California San Francisco, United States

Received: June 4, 2019

DOI: $10.5430 /$ jnep.v10n4p98
Accepted: December 9, 2019 Online Published: December 23, 2019

URL: https://doi.org/10.5430/jnep.v10n4p98

\begin{abstract}
The global age standardized prevalence of type 2 diabetes (T2DM) has doubled (4.7\% to $8.5 \%)$ over the last three decades and is increasing more rapidly in low and middle-income countries (LMICs). The global economic burden of diabetes affects individuals and health care systems and is estimated to cost $\$ 825$ billion USD a year. Within Mexico, T2DM is the second leading cause of mortality and the leading cause of morbidity using disability associated life years (DALYs). A retrospective chart review and cost analysis, analyzing those at risk of diabetes, was conducted at a rural community health clinic in Jalisco, Mexico. The goal was to project the cost of providing an appropriate scope of care and plan prevention-based population health programs. The results demonstrated that out of 264 charts reviewed, 218 (83\%) had one or more diabetic risk factor. The estimated per patient per visit cost is $\$ 127.22 \mathrm{MP}$ (Mexican Peso, 2018) and as the number of diabetes risk factors increases for an individual patient, the mean cost of their care to the system increases $(p<.001)$. Those with at least one risk factor comprise the majority in both males and females with a median age of 36 and median BMI of 28, and this group also has the highest percentage of borderline hypertension (46\%). This data demonstrates an opportunity to intervene in a group of young adults (ages 27-46) with a cluster of high-risk borderline risk factors and preventing them from developing obesity, hypertension and diabetes later in life.
\end{abstract}

Key Words: Mexico, Nursing, Global health, Community health, Diabetes, Cost analysis

\section{INTRODUCTION}

The global age-standardized prevalence of Type 2 Diabetes (T2DM) has doubled from $4.7 \%$ to $8.5 \%$ over the last three decades and is increasing more rapidly in low and middleincome countries (LMICs). ${ }^{[1,2]}$ The World Health Organization (WHO) estimates that by 2030, T2DM will increase from the eighth leading cause of disease to the fifth. ${ }^{[3]}$ Onset of the disease and its associated complications commonly occurs in the economically productive period of individuals' lives, thus financially affecting the economy of a country and the lives of individual families. ${ }^{[4]}$ The global economic burden of T2DM is estimated to cost $\$ 825$ billion US dollar (USD) a year. ${ }^{[5]}$ Due to higher prevalence rates in comparison with high income and upper middle income countries, LMICs carry a larger proportion of the financial and mortality burden of this growing epidemic. ${ }^{[2]}$ The complexity of the T2DM continuum of care is expensive to a healthcare system, and interventions focused on diabetes prevention have the potential to improve health outcomes for the population and reduce costs to the health care system. However, there are

*Correspondence: Katie Morales; Email: moralekn@gmail.com; Address: University of California San Francisco, United States. 
limited studies analyzing the costs to healthcare systems of caring for those with T2DM, as well as those at risk for the disease in LMICs. ${ }^{[4,6]}$ Understanding the profile of a population's prevalence and risk of diabetes and projecting the cost of providing an appropriate scope of care is useful for the planning of prevention-based population health programs.

\subsection{Burden of T2DM in Mexico}

The burden of disease in Mexico is primarily due to noncommunicable diseases (NCDs). T2DM and chronic kidney disease are two of the leading causes of mortality in Mexico following ischemic heart disease. ${ }^{[7]}$ Importantly, of the over 80 million people in Mexico, approximately 12 million $\left(14.8 \%\right.$ ) have diabetes. ${ }^{[8]}$ Among the Organization for Economic Cooperation and Development (OECD) countries, Mexico has the highest rates of hospital admissions due to uncontrolled diabetes, nearly twice the OECD average. In addition, diabetes is responsible for $14 \%$ of all deaths in Mexico, more than cancer, injuries, communicable disease, and respiratory disease. ${ }^{[9]}$ Only a quarter of people living in Mexico with diabetes are believed to have adequate blood sugar control. Further, it is projected that there are many others who are undiagnosed and unaware they have the disease. ${ }^{[10]}$ Chronic kidney disease is a complication of uncontrolled diabetes and, if left untreated, can progress to end stage renal disease and the need for lifelong hemodialysis or kidney transplantation. Mexico ranks first globally in incidence of chronic kidney disease and sixth in prevalence. Approximately $63 \%$ of those with end stage renal disease and on hemodialysis are due to T2DM. ${ }^{[11]}$ In 2011, the total direct cost of diabetes to Mexico's health care system was estimated at 3.4 billion USD. ${ }^{[12]}$

\subsection{Role of primary care in non-communicable disease screening and prevention}

Primary care provides a key role in the prevention, early detection and management of NCDs and associated risk factors. Screening at-risk individuals is particularly important, as NCDs can often be asymptomatic leading to a high burden of undiagnosed disease within the community. The Secretary of Health in Mexico provides T2DM screening recommendations in its Policy on Prevention, Treatment, and Control of Diabetes Mellitus. ${ }^{[13]}$ These policy guidelines recommend screening asymptomatic patients every three years starting at age 20 or at the onset of puberty when the following risk factors are present: obesity, sedentary lifestyle, a first degree relative with diabetes, history of gestational diabetes or polycystic ovarian syndrome, blood pressure greater or equal to 140/90, dyslipidemia, cardiovascular disease and those on antipsychotics. ${ }^{[13]}$ Screening asymptomatic individuals is essential to prevent late presentations with advanced

Published by Sciedu Press multi-system complications. ${ }^{[14]}$

Improving access to preventative medical care is key for decreasing the burden of NCDs, particularly diabetes. ${ }^{[15]}$ Therefore, the objectives of this study were: 1) to estimate risk for developing T2DM in a rural Mexican population, and 2) to estimate the cost of caring for patients at risk for diabetes compared to patients without risk. The overall goal of this work was to quantify cost of caring for patients with diabetes and the disease burden to engage funders and key stakeholders in program planning and evaluation. This study does not address the costs of interventions to existing practices to improve T2DM prevention and care.

\subsection{Setting}

Tiopa community health clinic is located in Autlan de Navarro, Jalisco Mexico. It provides adult and pediatric outpatient medical care to under-served and low-income families. On average, there are about 1,300-1,500 patient visits monthly. Services available include medical, dental, nutrition, psychology and dialysis. Medical visits are conducted on a walk-in basis and primarily used as an urgent care center. The Tiopa organization is a private, non-profit organization, and operates independently from the state of Jalisco's public health system.

\subsection{Significance of a chart review and cost analysis}

An external team of global health nursing researchers conducted a retrospective chart review and cost analysis of diabetes preventative care services at Tiopa community clinic in 2018. We predicted higher costs for patients with more risk factors and the clinics focus on urgent care. The aim of this paper is to describe the methodology used for conducting a cost analysis, the utility of the process, and the results obtained for informing and evaluating diabetes prevention program planning.

\section{METHOD}

\subsection{Data collection: Chart review}

Using the clinic registration database, a convenience sample of the first 50 adult charts entered for the months of February, March, April and May were accessed. The reason for visits varied and were not specific to diabetes screening. If charts were not able to be located or lacked documentation, the next charts entered for the month were accessed. The official Mexican national Policy on Prevention, Treatment, and Control of Diabetes Mellitus was used to identify which variables to collect based on the risk factors for diabetes relevant to this population. ${ }^{[13]}$ Risk factors for diabetes included: overweight (BMI $\geq 25$ ) or obese (BMI $\geq 30$ ), family history of diabetes, age greater or equal to 45 , and personal history 
of hypertension or dyslipidemia. Additional data collected included: diagnosis of diabetes or cardiovascular disease, documentation of lifestyle counseling or nutrition consult, medications and lab tests conducted. These data were entered into an Excel spreadsheet.

\subsection{Sample: Study subjects}

It was calculated that 186 medical charts needed to be reviewed based on $80 \%$ power to capture a $\$ 10 \mathrm{MP}$ difference in cost of care, a difference determined significant by clinical administrators. In order to capture the minimum of 186 charts of patients at risk of diabetes and account for potential issues derived from missing data, 264 charts were reviewed. Eligibility included all adults 18 and older who had utilized health-care services at the clinic within the last two years. Patients over 18 years of age are considered adults and the study was interested in the adult population. Exclusion criteria included charts with no documented vital signs, clinic visit greater than two years ago, patient $<18$ years. Dialysis patients were also excluded from this analysis due to the established high cost and frequency of visits, since the study focus was on early disease and prevention. Those patients with an established diabetes diagnosis, but were not receiving dialysis treatment, were included to analysze the cost for providing medical services to people with diabetes.

\subsection{Cost Analysis: Perspective}

For the cost analysis component, the cost of providing care to patients at risk of diabetes was done from a health care system perspective. The health care system perspective takes into account only those costs incurred by the clinic, as opposed to a societal perspective, which takes into account both patient and health system costs.

\subsection{Cost Analysis: Time and Motion}

The first step in the cost analysis process was to perform a time and motion study: a system of quantifying employee time and resource utilization. This was done through direct observation of staff but may also be done with selfadministered forms. For the purpose of this study, the time and motion portion was conducted through direct observation as staff forms were deemed burdensome by administration. Two different staff members with the same title (i.e. two nurses, two doctors etc.) were monitored over the course of an entire shift and data were collected on number of patients seen, amount of time spent with each patient and resource utilization. To gather variations in efficiency of practice, one experienced nurse as well as a new nurse were observed. Providers were observed during a day and evening shift to account for busier times of day.

\subsection{Cost Analysis: Data collection}

Utilizing accounting records, monthly staff salary and cost of supplies and infrastructure were collected. These costs were used to determine a general per patient per visit cost. This cost was assigned to all patients. An estimate of staff cost per patient was determined by estimating the time staff spent with each patient, such as registration, nursing and medical consultations, using the time and motion study (see Table 2). Based on this information, an employee's monthly salary per visit per patient cost was then generated. For those with indirect patient contact (cleaning staff, administration etc.) monthly income was divided by the average number of patients seen in one month. Although social work had direct patient contact, they saw patients that opted-in to use this service and would see these self-selected patients only at intake therefore their time was not a per visit expense.

To determine additional costs, other utilization data were extracted from the chart review including medications, diagnostic and clinical labs (including point of care testing) and nutrition consults. Registration dispenses medications and accepts the payment after patient visits, enabling the team to identify patients who obtained medication directly from Tiopa. For those that may have purchased medication at an outside clinic, the cost was estimated by obtaining the price of the medication from a local pharmacy. If lab results were in the chart, it was assumed that they were completed at Tiopa, unless the lab slip indicated otherwise (there were approximately five charts with labs done at another medical facility, a cost which was not included in the analysis). The individual costs incurred by each patient were then added to an Excel database.

\subsection{Data analysis}

Data analysis was conducted with $\mathrm{R}$ statistical software (version 1.0.153). ${ }^{[16]}$ To compare those with risk factors for diabetes and continuous variables such as age or cost, none of which were normally distributed, we used the Kruskal Wallis test. To compare categorical risk factors such as sex, the non-parametric Fisher's exact test was used to account for cells with a count of zero.

\section{RESULTS}

\subsection{Retrospective Chart Review}

All 264 charts reviewed had adequate data for the study analysis and therefore were included. Table 1 describes the demographics of the sample. The majority were female $66 \%$ $(n=175)$. Sixty percent $(n=157)$ of patients were categorized as overweight or obese. Those with a family history of T2DM comprised $12.5 \%$ of the sample population and $27 \%$ had a mixed family history that included a combination of 
T2DM, hypertension and cardiovascular disease. Personal history of diagnosed comorbidities were as follows: diabetes $(13 \%, \mathrm{n}=34)$, dyslipidemia $(9.5 \%, \mathrm{n}=25)$, and hypertension $(21 \%, \mathrm{n}=53)$.

Table 1. Sample population demographics

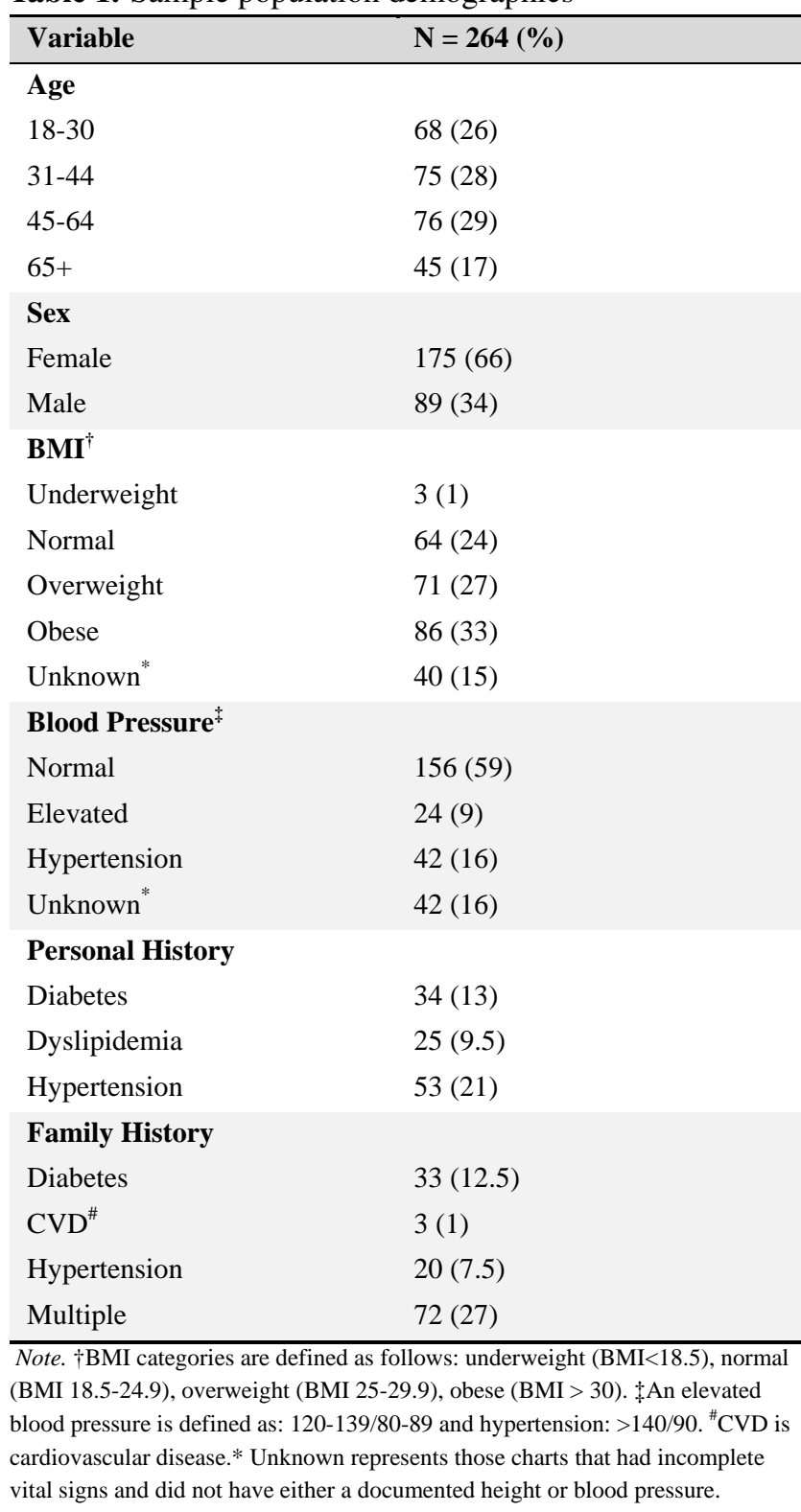

\subsection{Cost analysis}

Table 3 aggregates total costs to give the total per patient per visit cost. To account for the variation of costs for different patients, the cost of additional resources, such as medications, lab work and nutrition consults for individual patients, was added into the model. In summary, the base cost was the cost of: personnel + infrastructure + recurrent and capital goods $=$ per patient, per visit cost.

The estimated per patient per visit cost from a health care Published by Sciedu Press system perspective was $\$ 127.22$ Mexican Pesos (MP, 2018).

In Table 4, the varying costs were analyzed based on different levels of diabetes risk factors. The median per visit cost remained mostly stable across risk factors, with the exception of three risk factors that had a median cost of \$153.7 MP. The trend of the interquartile range increased with number of risk factors $(p<.001)$. Median cost demonstrates that the four risk factor group costs nearly as much as the no risk factor group. The mean cost per patient per visit shows a larger difference between groups, as it shows the zero risk factor group cost $\$ 148.4 \mathrm{MP}$ and the four risk factor group at $\$ 223.3 \mathrm{MP}$.

To assess the impact of uncertainty in the cost analysis, a one-way sensitivity analysis was conducted for key parameters. In Figure 1, the variables on the y-axis represent cost inputs used to determine the per visit per patient cost. Note that the base case value is $\$ 127.22$. Cost inputs were varied based on the range of uncertainty of the value. As the number of patients seen monthly increased, cost decreased. For all other inputs, such as provider time and equipment, an increase in these variables equated to an increase in cost. The two variables that had the largest impact on per patient cost were the number of patients seen monthly and fixed monthly costs.

Variable monthly costs: include office supplies and maintenance, costs that do not necessarily occur on a scheduled monthly basis. Fixed monthly costs: utilities, taxes. Nondirect clinic staff: accounting, administration, cleaning staff. Patients seen monthly: average number of patients seen monthly (1353). Cost of non-direct clinic staff, fixed and variable monthly costs, recurrent and capital goods were divided by this number to determine per patient per visit cost. Equipment: supplies such as gauze, gloves, needles etc. Nurse time: each patient is triaged by nurse and has vital signs taken, this is the estimated time in minutes. Doctor time: estimated time in minutes spent in consult. Base case value: $\$ 127.22$.

\section{Discussion}

Data from the 2016 National Health and Nutrition Survey (ENSANUT) in Mexico showed that $70 \%$ of adults were overweight or obese, diabetes prevalence was $9.4 \%$ and hypertension prevalence was $25.5 \% .{ }^{[17,18]}$ This study findings follow a similar trend with $60 \%$ of patients documented as overweight or obese, and hypertension prevalence of $21 \%$. However, this study found a higher rate of diabetes prevalence $(13 \%)$ and in a younger population. While risk is generally noted to begin at age 45 , this study did not have access to additional patient information that may have illuminated unusual risk factors for an earlier age at onset. It 
should be noted that this reflects documented and diagnosed disease and does not account for undiagnosed cases at Tiopa. It is likely that the true prevalence of diabetes among Tiopa

Table 2. Cost of staff with direct patient contact (\$Mexican Pesos, 2018)

\begin{tabular}{lllll}
\hline Resource & Daily work hours & $\begin{array}{l}\text { Average time in } \\
\text { minutes/per patient }\end{array}$ & Monthly salary & Cost per patient \\
\hline Physician & 6.5 & 10 & $\$ 11333$ & 12.1 \\
Nurse & 6 & 5 & $\$ 5308$ & 3.4 \\
Registration/" pharmacy & 8 & 2 & $\$ 6056$ & 1.3 \\
Nutritionist (first visit) & 8 & 60 & $\$ 5308$ & 33.2 \\
Nutritionist (subsequent visits) & 8 & 30 & $\$ 5308$ & 16.6 \\
Social Worker $^{\dagger}$ & 8 & 17.5 & $\$ 4694$ & 8.6
\end{tabular}

Note. $^{*}$ Registration also serves as pharmacy, dispensing medications after the medical appointment. ${ }^{\dagger}$ Patients opt-in to see social work to register as patients and determine cost of visits, which is a sliding scale based on income.

Table 3. Total cost to the health care system (\$Mexican Pesos, 2018/patient/visit)

\begin{tabular}{|c|c|c|}
\hline Resource & Amount & $\begin{array}{l}\text { Per patient } \\
\text { cost }(\$ M P)\end{array}$ \\
\hline \multicolumn{3}{|l|}{ Personnel } \\
\hline Physician & 1 & 12.1 \\
\hline Nurse & 1 & 3.4 \\
\hline Registration/pharmacy & 1 & 1.3 \\
\hline Other (admin and cleaning staff) ${ }^{*}$ & 6 & 32.99 \\
\hline \multicolumn{3}{|l|}{ Infrastructure } \\
\hline $\operatorname{Rent}^{\dagger}$ & $\mathrm{n} / \mathrm{a}$ & $\$ 0.00$ \\
\hline Fixed monthly costs (utilities, taxes) & $\mathrm{n} / \mathrm{a}$ & $\$ 61.95$ \\
\hline Variable costs (office supplies, maintenance) & $\mathrm{n} / \mathrm{a}$ & $\$ 8.45$ \\
\hline \multicolumn{3}{|l|}{ Recurrent goods } \\
\hline Equipment ${ }^{\ddagger}$ & & $\$ 6.39$ \\
\hline \multicolumn{3}{|l|}{ Capital goods } \\
\hline Blood pressure machine & 1 & $\$ 0.32$ \\
\hline Accucheck $^{\#}$ & 1 & $\$ 0.00$ \\
\hline Scale & 1 & $\$ 0.32$ \\
\hline TOTAL & & $\$ 127.22$ \\
\hline \multicolumn{3}{|c|}{$\begin{array}{l}\text { Note. }{ }^{*} \text { This cost included administration, accounting, cleaning staff, social work } \\
\text { and excluded those with recurrent direct patient contact. }{ }^{\dagger} \text { Rent is zero for this } \\
\text { clinic. }{ }^{\star} \text { Equipment includes recurrent medical supplies such as gauze, gloves, lab } \\
\text { draw equipment etc. }{ }^{*} \text { Accucheck is a blood sugar point-of-care device that was } \\
\text { donated by a local private entity. }\end{array}$} \\
\hline
\end{tabular}

The findings from this study show that $83 \%$ of patients had at least one risk factor for diabetes, with a median age of 36 (IQR 27-46), and median BMI in the overweight classification of 28. Those with one risk factor were also more likely to have elevated blood pressure $(46 \%)$. While those patients were not necessarily diagnosed with hypertension, they were found to be borderline hypertensive at their most recent appointment. The characteristics of this group present a key opportunity to intervene with at-risk young adults to prevent them from developing obesity, hypertension and diabetes in the future. ${ }^{[14]}$ Those patients with one diabetic risk factor are a key cohort with which to begin diabetic prevention strategies. Addressing lifestyle modifications that can lower BMI and blood pressure in patients ages 27- 46 years may slow the progression to diabetes and hypertension. ${ }^{[14]}$ Patients in this setting would benefit from individualized counseling and reinforcement of lifestyle changes to prevent progression of disease. While agreeing upon an ideal HbA1c would be ideal, lab capacity in low resource settings may be limited, making other indicators of disease more important for monitoring disease development or progression. ${ }^{[20]}$

Table 4. Total cost by risk factor and diabetes diagnosis (\$Mexican Pesos, 2018/patient/visit)

\begin{tabular}{llllllll}
\hline Cost $^{*}$ & $\begin{array}{l}\text { No risk } \\
\text { factors } \\
\mathbf{n = 4 6}\end{array}$ & $\begin{array}{l}\mathbf{1} \text { risk factor } \\
\mathbf{n}=\mathbf{9 6}\end{array}$ & $\begin{array}{l}\mathbf{2} \text { risk factors } \\
\mathbf{n = 7 0}\end{array}$ & $\begin{array}{l}\mathbf{3} \text { risk factors } \\
\mathbf{n = 4 4}\end{array}$ & $\begin{array}{l}\mathbf{4} \text { risk factors } \\
\mathbf{n = 8}\end{array}$ & $\begin{array}{l}\text { No diabetes } \\
\text { diagnoses } \\
\mathbf{n}=\mathbf{2 3 0}\end{array}$ & $\begin{array}{l}\text { Diagnosed } \\
\text { diabetes } \\
\mathbf{n}=\mathbf{3 4}\end{array}$ \\
\hline Median & 127.2 & 127.2 & 127.2 & 153.7 & 136.1 & 127.2 & 164 \\
$(\mathrm{IQR})$ & $(127.2-127.2)$ & $(127.2-141.3)$ & $(127.2-144)$ & $(127.2-266.7)$ & $(127.2-331.7)$ & $(127.2-142.4)$ & $(130.7-353.2)$ \\
Mean & 148.4 & 152.2 & 160.3 & 209.3 & 223.3 & 155.8 & 230 \\
$p$-value & $<.001$ & & & & & & $<.001$ \\
\hline
\end{tabular}

*Cost reflects the total cost to the health care system including; base case cost of \$127.2, labs, medications, and nutrition visits. 


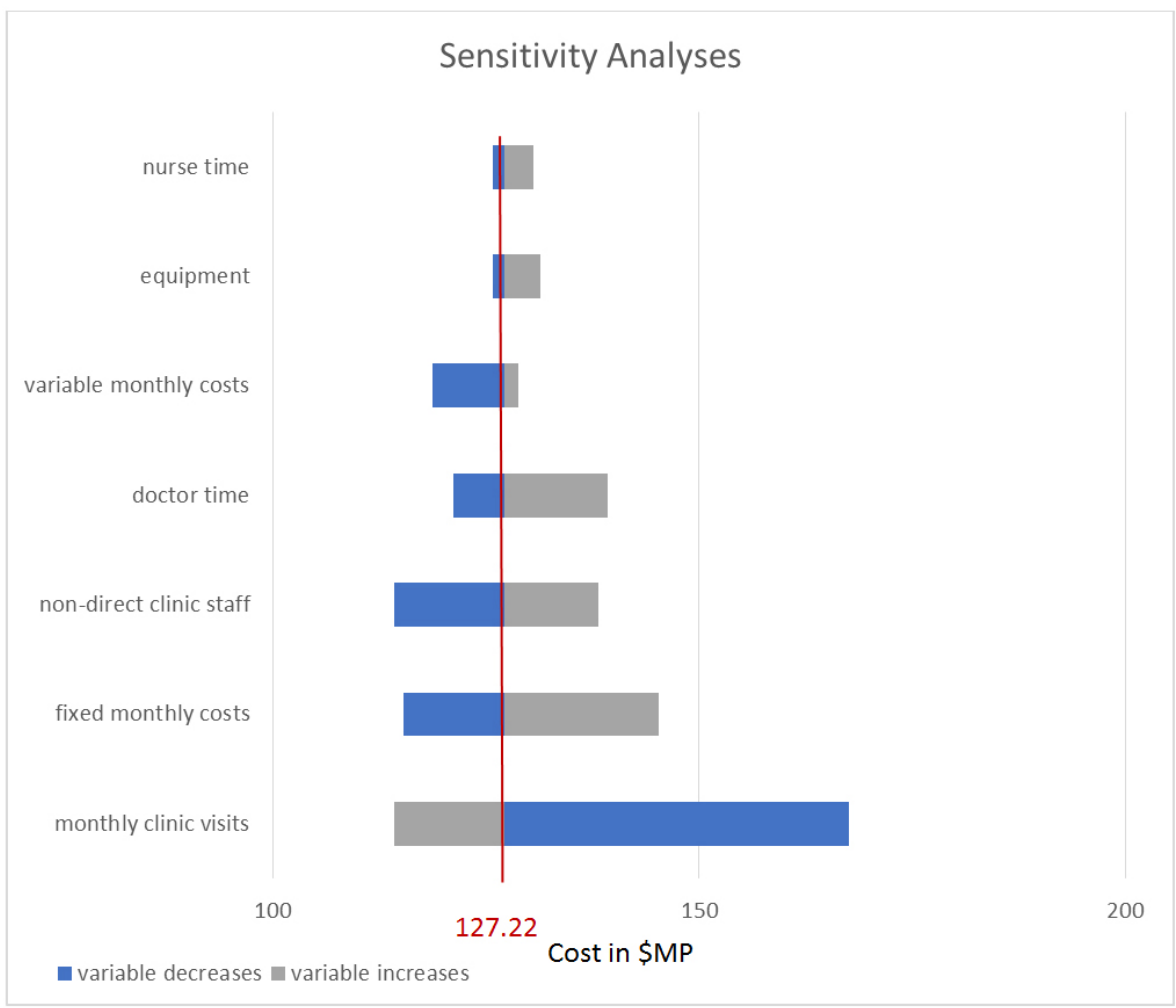

Figure 1. Managers' and nurses' competence assessments

The cost analysis demonstrated that while, on average, patients that present to Tiopa cost the healthcare system $\$ 127.22 \mathrm{MP}$, that cost increases by $\$ 36.8$ per visit for patients with diabetes. This is an expected finding, as people with diabetes may have more frequent lab work and are likely to be using more medications in comparison to individuals who do not have diabetes. In 2012, ENSANUT found that a patient with diabetes costs the Mexican health care system on average $\$ 707$ MP per year. ${ }^{[17]}$ These costs could potentially be avoided by early intervention. This analysis also showed that the cost of patients who are at-risk for diabetes increases as number of risk factors increases and is highest for those in the three-risk factor category. This trend may be because $64 \%$ of those in the three-risk factor category have diagnosed diabetes. As patients go from one risk factor to two risk factors, the diabetic prevalence increases from $4 \%$ to $63 \%$. This demonstrates the importance of focusing on the one risk factor group (pre-diabetes) for prevention strategies. It was noted that the four risk factor group had costs similar to the group with no risk factors. This may be due to the small sample size in this group $(n=8)$ or perhaps these patients are sicker and receive additional care at a tertiary health center and come to Tiopa for urgent care visits only. Understanding the financial impact of disease burden on health care systems is essential to financial planning and sustainability of diabetes prevention programs. ${ }^{[19]}$
The sensitivity analysis found that increasing the number of monthly patient visits decreases cost, as this disperses costs and resources across a larger pool of patients. Maximizing providers' time and ensuring that they are seeing a full schedule of patients may improve costs, however, it may not be practical in the study setting. It is also possible that having a dedicated nurse or nursing staff to see patients at risk for diabetes may lower costs, given the number of patients seen and the ability of nurses to provide comprehensive and adequate care and counseling. ${ }^{[21]}$

There are several limitations in this study, the first being recall bias. Data extracted from chart reviews relies partially on patient reported data for family and personal history. This may have led to an over or underestimate of the true prevalence of certain risk factors. It should also be noted that the estimates are capturing only those in care at Tiopa and not undiagnosed disease in the community. The second limitation is selection bias. Data was obtained from one clinic in Autlan and, therefore, findings may not be generalizable to the entire Autlan population. Only services provided by Tiopa and corresponding costs were included, findings may not be generalized to other clinics. The goal of this study, however, was to provide key Tiopa stakeholders with information to identify areas for early intervention. In addition, this study may offer tools for other institutions who wish to 
extract similar data from their respective facilities.

While this analysis looked at the charts of patients at risk of diabetes, depending on local disease burden, any chronic disease could be analyzed for financial planning and forecasting. ${ }^{[19]}$ Identifying and quantifying those patients at risk for chronic, preventable disease is necessary for lowering the chronic disease burden and its sequelae. From a financial perspective, a cost analysis can justify the need for continued financial support to funders and key stakeholders.

In summary, our study showed that the cost of providing care in this primary care setting increased as patients' risk factors for diabetes increased. Primary care clinics are the ideal setting for disease prevention and intervention before the development of diabetes. Given the burden of T2DM in Mexico and globally there is a need for more effective primary care disease prevention strategies, to not only reduce health care costs but to improve patient outcomes. A continuation of this study could compare costs of diabetes preventative programs to the current costs of caring for patients with diabetes. Future studies could estimate the costs of specific disease prevention programs.

\section{CONFLiCTS OF INTEREST Disclosure}

The authors declare that there is no conflict of interest statement.

\section{REFERENCES}

[1] Mathers CD, Loncar D. Projections of Global Mortality and Burden of Disease from 2002 to 2030. PLoS Med. 2006; 3: e442. PMid:17132052 https://doi.org/10.1371/journal.pmed.0 030442

[2] Dagenais GR, Gerstein HC, Zhang X, et al. Variations in Diabetes Prevalence in Low-, Middle-, and High-Income Countries: Results From the Prospective Urban and Rural Epidemiological Study. Diabetes Care. 2016; 39: 780-7. PMid:26965719 https: //doi.org/10.2337/dc15-2338

[3] GLOBAL REPORT ON DIABETES WHO Library Cataloguing-inPublication Data. ISBN. 1978: 92-4.

[4] WHO. The prevention of diabetes and its complications. WHO 2011. Available from: http://www. who.int/diabetes/preventionf lyer/en/

[5] Risk Factor Collaboration N. Worldwide trends in diabetes since 1980: a pooled analysis of 751 population-based studies with $4 \bullet 4$ million participants NCD Risk Factor Collaboration (NCD-RisC)*. Lancet. 2016; 387: 1513-30.

[6] Seuring T, Archangelidi O, Suhrcke M. The Economic Costs of Type 2 Diabetes: A Global Systematic Review. Pharmacoeconomics. 2015; 33: 811-31. PMid:25787932 https://doi.org/10.1007/ s40273-015-0268-9

[7] IHME. GBD Compare, IHME Viz Hub. Available from: https: //vizhub.healthdata.org/gbd-compare/

[8] IDF North America and Caribbean members: Mexico. International Diabetes Federation. 2019. Available from: https://www.idf.org/our-network/regions-members/n orth-america-and-caribbean/members/66-mexico.html

[9] WHO. Country Profile: Mexico. 2016. Available from: https: //www.who.int/diabetes/country-profiles/mex_en.pdf

[10] González-Pier E, Barraza-Lloréns M, Beyeler N, et al. Articles Mexico's path towards the Sustainable Development Goal for health: an assessment of the feasibility of reducing premature mortality by $40 \%$ by 2030. 2016. https://doi .org/10.1016/S2214-109X (16) 3 0181-4

[11] Solis-Vargas E, Evangelista-Carrillo LA, Puentes-Camacho A, et al. Epidemiological Characteristics of the Largest Kidney Transplant Program in Mexico: Western National Medical Center, Mexican Institute of Social Security. Transplant Proc. 2016; 48: 1999-2005.
PMid:27569935 https://doi.org/10.1016/j.transproceed .2016 .03 .039

[12] Arredondo A, Reyes G. Health disparities from economic burden of diabetes in middle-income countries: evidence from México. PLoS One. 2013; 8: e68443. PMid:23874629 https://doi.org/10.1 371/journal. pone. 0068443

[13] Hernandez Avila M. NORMA Oficial Mexicana NOM-015-SSA22010, Para la prevención, tratamiento y control de la diabetes mellitus. Secr. Salud. Available from: http://www . dof .gob.mx/normas0 ficiales/4215/salud/salud.htm

[14] WHO. Noncommunicable diseases. WHO 2017. Available from: ht tp://www. who.int/mediacentre/factsheets/fs355/en/

[15] Oxford Business Group. Public-private cooperation increases in Mexico's health sector, Mexico 2015. xford Business Group. Available from: https://oxfordbusinessgroup.com/overvi ew/healthy-prospects-cooperation-between-public-a nd-private-sectors-increasing

[16] The R Project for Statistical Computing. Available from: https: //www.r-project.org/

[17] Instituto Nacional de Salud Publica. Encuesta Nacional de Salud y Nutricion. 2012. Available from: https://ensanut.insp.mx/in formes/ENSANUT2012ResultadosNacionales.pdf

[18] Observatorio Mexicano de Enfermedades No Transmisibles. Latest diabetes statistics in Mexico-ENSANUT 2016. OMENT. 2016. Available from: http://oment.uanl.mx/latest-diabetes-stati stics-in-mexico-ensanut-2016/?lang=en

[19] Global Burden of Disease Health Financing Collaborator Network JL, Campbell M, Chapin A, et al. Future and potential spending on health 2015-40: development assistance for health, and government, prepaid private, and out-of-pocket health spending in 184 countries. Lancet (London, England). 2017; 389: 2005-30.

[20] Type 2 diabetes in adults: management NICE guideline [NG28]. 2019. Available from: https://www.nice.org.uk/guidance/n g28/chapter/1-Recommendations

[21] Golden SH, Maruthr N, Mathioudakis N, et al. The Case for Diabetes Population Health Improvement: Evidence-Based Programming for Population Outcomes in Diabetes. Curr Diabetes Reports. 2017; 17: 51. PMid:28567711 https ://doi .org/10.1007/s11892-0 17-0875-2 